University of Nebraska - Lincoln

DigitalCommons@University of Nebraska - Lincoln

6-9-2006

\title{
High-Resolution Thin-Film Device to Sense Texture by Touch
}

Vivek C. Maheshwari

University of Nebraska-Lincoln, vmaheshw@uwaterloo.ca

Ravi F. Saraf

University of Nebraska-Lincoln, rsaraf2@unl.edu

Follow this and additional works at: https://digitalcommons.unl.edu/cbmesaraf

Part of the Biomechanics and Biotransport Commons

Maheshwari, Vivek C. and Saraf, Ravi F., "High-Resolution Thin-Film Device to Sense Texture by Touch" (2006). Ravi Saraf Publications. 8.

https://digitalcommons.unl.edu/cbmesaraf/8

This Article is brought to you for free and open access by the Chemical and Biomolecular Research Papers -- Faculty Authors Series at DigitalCommons@University of Nebraska - Lincoln. It has been accepted for inclusion in Ravi Saraf Publications by an authorized administrator of DigitalCommons@University of Nebraska - Lincoln. 


\section{High-Resolution Thin-Film Device to Sense Texture by Touch}

\author{
Vivek Maheshwari ${ }^{1}$ and Ravi F. Saraf ${ }^{1,2 *}$
}

Touch (or tactile) sensors are gaining renewed interest as the level of sophistication in the application of minimum invasive surgery and humanoid robots increases. The spatial resolution of current large-area (greater than $1 \mathrm{~cm}^{2}$ ) tactile sensor lags by more than an order of magnitude compared with the human finger. By using metal and semiconducting nanoparticles, a 100-nm-thick, large-area thin-film device is self-assembled such that the change in current density through the film and the electroluminescent light intensity are linearly proportional to the local stress. A stress image is obtained by pressing a copper grid and a United States 1-cent coin on the device and focusing the resulting electroluminescent light directly on the charge-coupled device. Both the lateral and height resolution of texture are comparable to the human finger at similar stress levels of $\sim 10$ kilopascals.

$\mathrm{S}$ ensation of touch, primarily the determination of stress distribution over the area of physical contact between the sensor and the object surfaces (1), is a critical component to advance minimum invasive surgical procedures by giving the surgeon a "touch sensation" (2-6) to decipher, for example, cancer tissue (3) and gallstone (2) using the signal from tactile sensor (7). Moreover, there is great interest in developing humanoid robots $(8,9)$ that can sense shapes $(10,11)$, textures $(12,13)$, and hardness (14) and manipulate complex objects (11), which are not readily possible by vision alone. Touch (or tactile) sensors are usually made as micro-electromechanical systems composed of micromachined deformable components (15) or by integrating chip with electronic circuit and strain sensitive materials, such as magento-resistive ceramics (16), piezoelectric polymers $(17,18)$, and strain sensitive conducting elastomers $(19,20)$. Tactile sensors from optical data have been demonstrated in which the contact stress distribution is calculated from the change in shape of the deformable sensor surface obtained by a camera (21). For small-area devices, such as an 8 by 8 array of capacitance sensors, spatial resolution of 100 $\mu \mathrm{m}$ is demonstrated (22). However, for largearea devices, $\sim 1 \mathrm{~cm}^{2}$ or larger, the spatial resolution for stress distribution is, at best, in the $\sim 2 \mathrm{~mm}$ range (20) compared with the $\sim 40-\mu \mathrm{m}$ resolution achieved by the surface of a human finger (23).

We report a $\sim 100-\mathrm{nm}$-thick, $2.5-\mathrm{cm}^{2}$ device, based on the principle of electron tunneling, capable of imaging stress distribution with spatial resolution of $\sim 40 \mu \mathrm{m}$. The height resolution is $<5 \mu \mathrm{m}$, comparable to the $\sim 2 \mu \mathrm{m}$ for a human finger (24). Our device, composed of metal and semiconducting nanoparticles, directly converts local stress into electrolumi-

${ }^{1}$ Department of Chemical Engineering, University of Nebraska, Lincoln, NE 68588, USA. ${ }^{2}$ Edward Via Virginia College of Osteopathic Medicine, Blacksburg, VA 24060, USA.

*To whom correspondence should be addressed. E-mail: rsaraf@unlnotes.unl.edu nescent light and modulation in local current density. Both the electroluminescence intensity and current density are linearly proportional to the local stress.

To demonstrate the high-resolution capability, we image stress by directly focusing the electroluminescent light signal on a chargecoupled device (CCD) camera. The sensitivity or minimum stress required for direct imaging of touch on CCD is $\sim 9 \mathrm{kPa}$, which is well within the 10 to $40 \mathrm{kPa}$ range that a human finger applies to sense texture and shape (25). To demonstrate the spatial resolution of the device, we use a copper grid to hold the specimen for transmission electron microscope (TEM) analysis. When the $\mathrm{Cu}$ grid composed of free-standing $40-\mu \mathrm{m}$-wide lines at a pitch of $220 \mu \mathrm{m}$ is pressed on the sample, the stress distribution from the features produces spatial variation in electroluminescent light that is directly imaged on the CCD. Furthermore, we press a U.S. 1-cent coin against the device and image the stress distribution from the embossing on the coin directly onto the CCD. The fabrication requires no lithography, and the transduction of stress distribution is virtually continuous at 100-nm scale. A simple model based on electron tunneling between the nanoparticle layers and ionic conductivity through the dielectric medium explains the characteristics of the device.
The device (Fig. 1) consists of alternating layers of $\mathrm{Au}$ (10 nm thick) and $\mathrm{CdS}$ (3 nm thick) nanoparticles separated by dielectric layers (DL), composed of stacked alternating layers of poly(styrene sulfonate) (PSS) and poly(allylamine hydrochloride) (PAH). All the monolayers of polymer and nanoparticles are deposited from solution using layer-by-layer self-assembly $(26,27)$. The particular device described here has three $\mathrm{Au}$ layers and two CdS layers, with four layers each of PAH and PSS as the interlying DL [see details of structure and process in (28)]. The atomic force microscope (AFM) images in Fig. 1B indicate that the nanoparticles do not form long-range, (electrically) percolating clusters. Consistent with the AFM image, the film is insulating in the inplane direction, as independently confirmed by measuring two-point electrical continuity using spring-loaded flat $\mathrm{Au}$ (pogo) pins.

In the vertical direction, the film is conducting due to tunneling between the $\mathrm{Au}$ and $\mathrm{CdS}$ layers. Figure $2 \mathrm{~A}$ shows the current density $J$ through the film as a function of bias $V$ between the bottom ITO and top Au electrode under a uniform compressive stress $\sigma$. The uniform stress is applied by placing an optically flat quartz disk on the flexible $\mathrm{Au}$ electrode. The nonlinear $J-V$ curve is fit based on a model combining the field-assisted electron tunneling current [i.e., the Fowler-Nordheim equation (29-31)] through the nanoparticles and the ionic (leakage) current due to ions in the polyelectrolyte. According to the relation $J_{\text {tunneling }}=$ $P \exp (-a K / V)$, where, $a$ is the (vertical) interparticle distance, $K$ is a critical field for activated tunneling that depends on the work functions of the particles, and $P$ at constant temperature is proportional to $V^{2}$ and the number density of carriers for conduction $(29,32)$. The ionic leakage-current, $J_{\text {leakage }}=V / R$, is due to mobile ions $\left(\mathrm{H}^{+}, \mathrm{Na}^{+}\right.$and $\left.\mathrm{OH}^{-}, \mathrm{Cl}^{-}\right)$in the polyelectrolytes, where $R$, the ohmic resistance, is proportional to the distance between the electrodes. A three-parameter (i.e., $R, a K$, and $P$ ) fit for three sets of data, with corresponding straight line due to leakage $I-V$, is an excellent match to the experiment for the whole range of
Fig. 1. Multilayer structure of electrooptical device. (A) Schematic of the unit structure of the device showing nanoparticle monolayers spaced by an organic DL composed of polyelectrolytes PSS and PAH. A total of three $\mathrm{Au}$ and

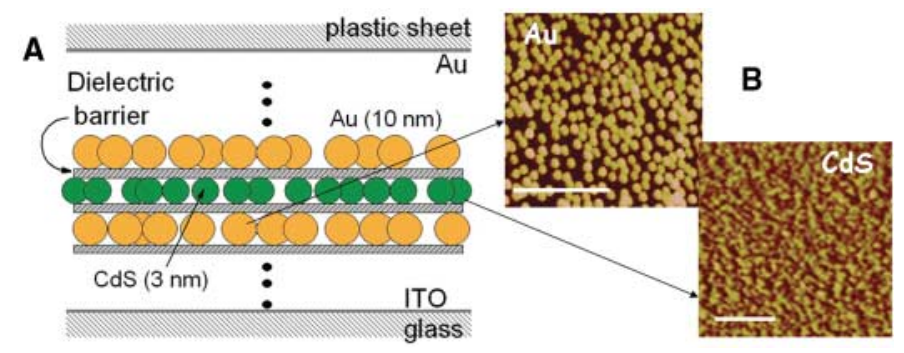
two CdS nanoparticle monolayers with dielectric barriers are deposited. The top Au electrode is coated with flexible plastic, and the bottom transparent Indium-Tin Oxide (ITO) electrode is on glass. The electroluminescent light from the CdS is measured through the bottom electrode. (B) AFM topography (i.e., height) image after deposition of first Au layer and the first CdS layer after that. The scale is $50 \mathrm{~nm}$. 
$V$ and $\sigma$ studied (Fig. 2A). The three fitting parameters are self-consistent as applied stress increases (Fig. 2B); $R$ from the fit decreases linearly with $\sigma$, implying that the film deforms linearly. The estimated resistivity of $\sim 1.6 \times 10^{8}$ $\Omega-\mathrm{m}$ is reasonable for ion conductivity in PSS and PAH under ambient humidity $(33,34)$. The linear decrease in $\mathrm{Ka}$ with $\sigma$ indicates that the interparticle spacing (i.e., $a$ ) also decreases linearly with $\sigma$, which is consistent with linear deformation of overall thickness. Assuming $a$ (at $\sigma=0) \sim 5 \mathrm{~nm}$, the critical field $K$ of $\sim 10^{9} \mathrm{~V} / \mathrm{m}$ is reasonable $(29,32)$. Because $P$ depends on the carrier density, at fixed $V$, the linear increase in $P$ may be attributed to a linear increase in the number of percolating channels between the electrodes as the film is deformed. Furthermore, although not shown, consistent with the FowlerNordheim equation, $P \sim V^{2}$ (at constant load) for the complete range of operating conditions $(>8 \mathrm{~V})$. The curve fit in Fig. 2A indicates that the tunneling process becomes important beyond $\sim 8 \mathrm{~V}$ (indicated as a box). Electroluminescence will occur only when tunneling through $\mathrm{CdS}$ occurs. Hence, the electroluminescence intensity $I_{\mathrm{EL}}$ should be measurable above the bias of $\sim 8 \pm 1 \mathrm{~V} . I_{\mathrm{EL}}$ as reported is intensity (in arbitrary units) per 16 by $16 \mu \mathrm{m}$ pixel of the CCD camera. The noise level based on imaging a blank sample (i.e., dark current) is 1 arbitrary unit. The $I_{\mathrm{EL}}$ threshold at $\sim 8 \mathrm{~V}$ further supports the chargetransport model (Fig. 2C). The average $I_{\mathrm{EL}}$ per pixel is limited to $80 \mathrm{kPa}$ because the electroluminescence from the edge of the disk is enhanced as a result of "square-edge-punch" stress concentration (35). Furthermore, as $J_{\text {tunneling }}$ increases exponentially with stress compared with a linear increase for $J_{\text {leakage, }}$ at larger bias (i.e., $>18 \mathrm{~V}$ ) tunneling dominates over leakage current. The deformation is reversible with nominal hysterisis (Fig. 2D).

To assess the capabilities of the device for measuring surface topography and texture, a TEM specimen holder from Electron Microscopy Sciences (i.e., a $\mathrm{Cu}$ grid) and a U.S. 1-cent coin was pressed against the flexible $\mathrm{Au}$ electrode of the device, and the resulting electroluminescent light from the device was focused on the CCD camera below the ITO/ glass substrate (Fig. 3A). The embossing of President Lincoln's head on the coin is apparent
A

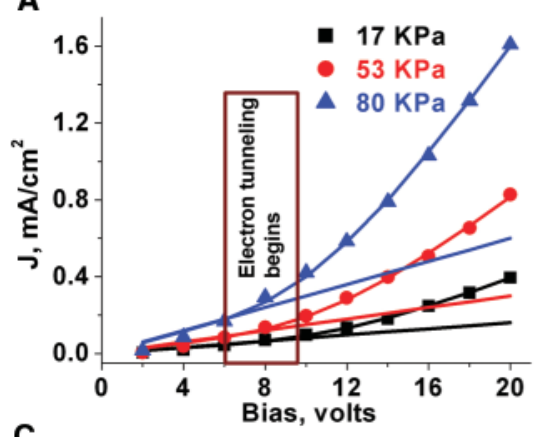

C

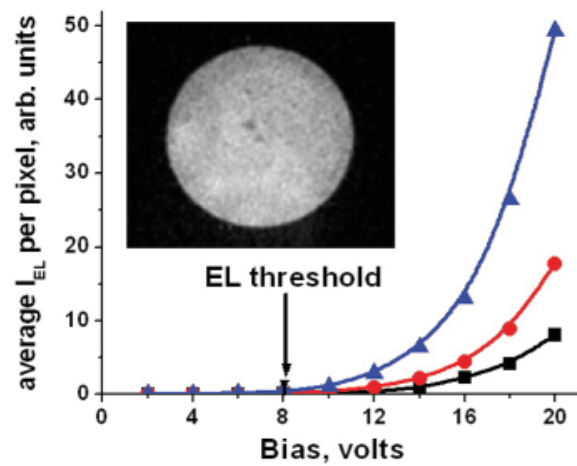

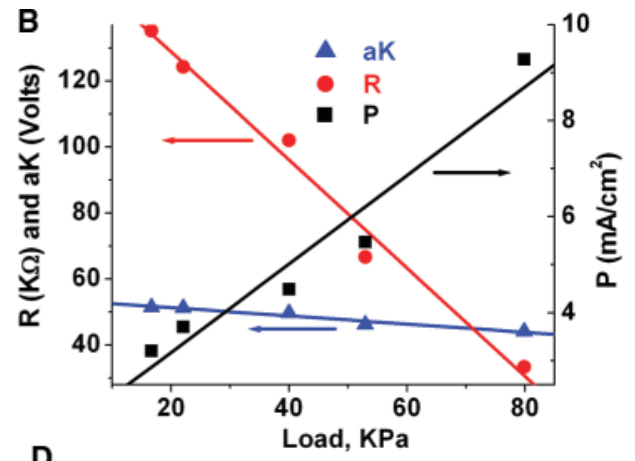

D

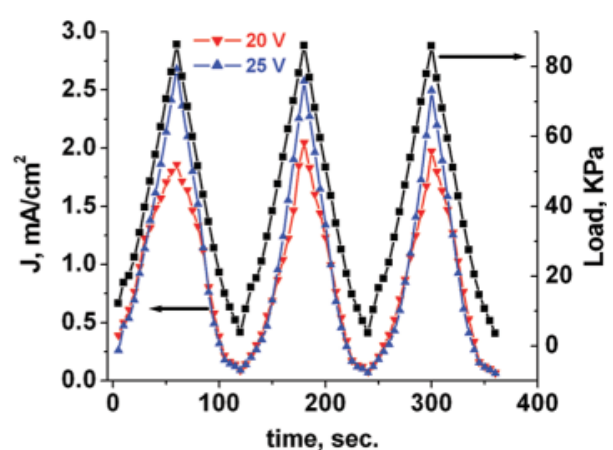

Fig. 2. Electro-optical characteristics of the device and electron-transport mechanism. The device is compressed using an optically flat quartz disk on the flexible Au electrode. (A) $J-V$ data points at various $\sigma$. The fitted line is based on a model combining charge transport by electron tunneling

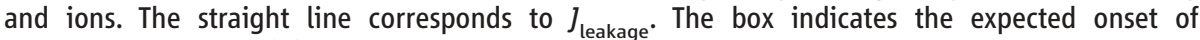
electroluminescence. (B) The three fitting parameters estimated from fitting the $J-V$ curve show linear dependence, indicating that the deformation is linear with respect to load. (C) The electroluminescence from the $\mathrm{CdS}$ nanoparticles for $\sigma$ in 17 to $80 \mathrm{kPa}$ range commences at $\sim 8 \mathrm{~V}$. The inset is the electroluminescence image on the CCD camera below the ITO electrode corresponding to the $1.1-\mathrm{cm}$-diameter quartz disk pressing the top electrode (Fig. 3A). Uniform light indicates uniform load distribution. The data symbols correspond to Fig. 2A. (D) The current J monitored under dynamic loading of the device at a fixed bias of 20 and $25 \mathrm{~V}$ indicates reasonably low hysteresis. The line is to guide the eye.

in the stress image obtained on the CCD. The overall intensity of the stress image increases with average stress $\sigma$ (Fig. 3A). The TEM grid lines have a trapezoidal cross-section with a top base of $40 \mu \mathrm{m}$ that is in contact with the device, a width of $80 \mu \mathrm{m}$ at half height, and interline spacing of $230 \mu \mathrm{m}$ (Fig. 3B, inset, and Fig. $3 \mathrm{C}$ ). Lines of the $\mathrm{Cu}$ grid (seen in the optical microscope image) are clearly resolved in the stress image (Fig. 3B). Comparison between the topography and $I_{\mathrm{EL}}$ scan of the TEM Cu grid shows that the flat surface of $40-\mu \mathrm{m}$ line width in contact with the device is well resolved, leading to a lateral spatial resolution of at least $40 \mu \mathrm{m}$.

The remarkable correspondence between the optical microscope image and the stress image seen in Fig. 3, A, D, and E, indicates that the variation in stress distribution caused by the embossing on a U.S. 1-cent coin leads to significant change in local current density, which in turn is manifested as modulation in $I_{\mathrm{EL}}$. The device can decipher fine features, such as wrinkles on the clothing of President Lincoln and the letters "TY" in "LIBERTY," observed in the optical microscope and the stress image (Fig. 3D). Because the electroluminescence intensity from the device is virtually continuous, the resolution of the stress image is determined by the optics and the CCD camera. For the CCD with a 512 by 512 array of $\sim 16-\mu \mathrm{m}$ pixels, the $I_{\mathrm{EL}}$ is well over noise level (Fig. 3, $\mathrm{C}, \mathrm{E}$, and $\mathrm{F}$ ), indicating that the electroluminescence is enough to achieve lateral spatial resolution of at least $\sim 20 \mu \mathrm{m}$ (as the area of chip and disk are comparable). However, the stress will be "smeared" as a result of the thickness of the flexible $\mathrm{Au}$ electrode, leading to higher resolution. Furthermore, from Fig. 3, E and F, height modulation of $\sim 10 \mu \mathrm{m}$ is measurable, indicating that the device is fairly deformable. The high compliance is perhaps due to some interpenetration among the nanoparticle layers. The inset in Fig. 3F shows that the embossing is rough at the micron scales. This local roughness will cause nonuniformity in the surface charge distribution, leading to "hot spots" of high electric field strength. The "pointillist" pressure images are attributed to these hot spots.

The relationship between the optical (i.e., $I_{\mathrm{EL}}$ ) and electronic (i.e., $J$ ) signal from the device pressed by the quartz disk is linear (Fig. 4A), implying that the stress image from either signal would be similar. Above, we have demonstrated the tactile sensor principle by measuring $I_{\mathrm{EL}}$. Extension to a fully electronic device by measuring $J$, required for certain applications (such as surgery), is possible using standard microelectronic circuitry. For example, an electronic device could be made by selfassembling the multilayer thin-film between mutually perpendicular parallel lines of $\mathrm{Au}$ (electrodes) on flexible plastic backing (i.e., flex-circuit). The stress distribution would be 
A

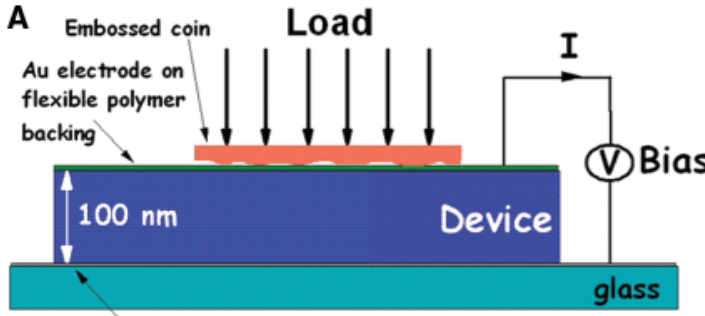

Transparent Electrode (ITO)

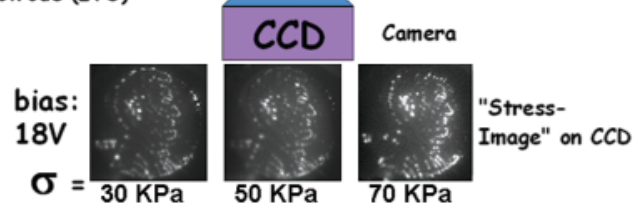

D

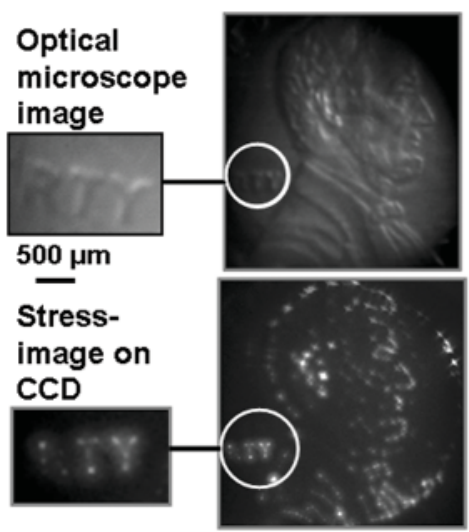

E

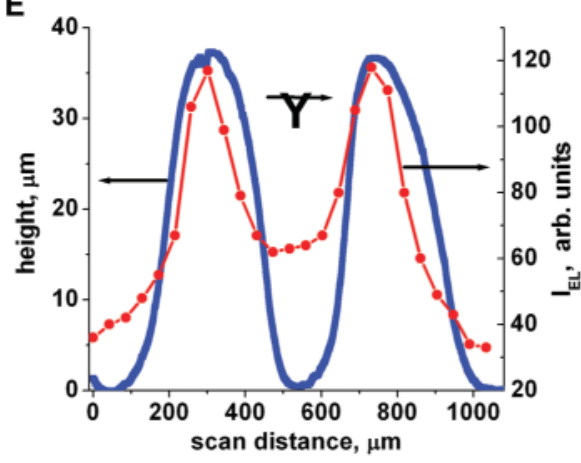

C

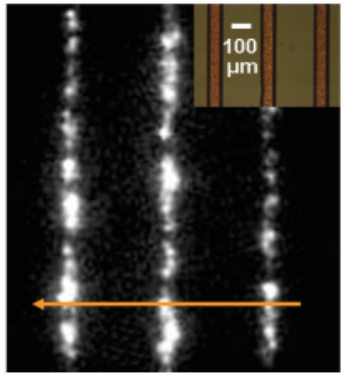

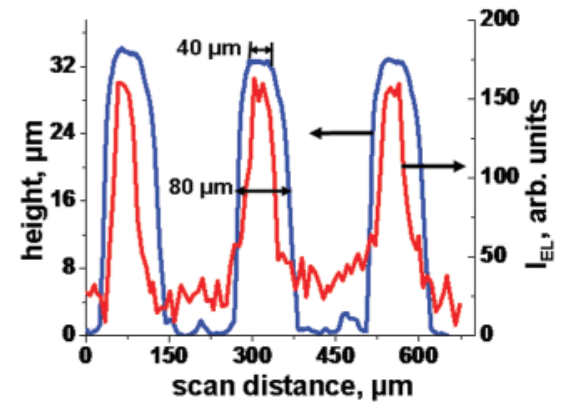

scan distance, $\mu \mathrm{m}$
$\mathbf{F}$

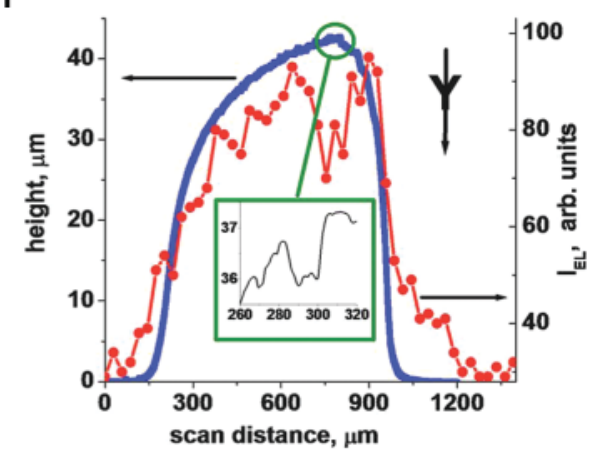

Fig. 3. Characteristics of the pressure imaging device. (A) Schematic of the setup to image texture of a metal coin; also shown are the pressure images taken on CCD camera at three different compressive stresses. The diameter of the coin is $\sim 2 \mathrm{~cm}$, and the device is a $2.5-\mathrm{cm}$ square. The bias between the coin and the ITO electrode is fixed at 18 V. (B) Comparison between optical microscope (inset) and pressure image (at $V=18 \mathrm{~V}$ and $\sigma=40 \mathrm{kPa}$ ) of a TEM Cu grid. The horizontal line in the pressure image is the $I_{\mathrm{EL}} \mathrm{scan}$ shown in Fig. 3C. (C) Comparison between the topographic and $I_{\mathrm{EL}}$ scans across the TEM Cu grid. (D) Comparison between optical microscope and pressure image (at $V=18 \mathrm{~V}$ and $\sigma=30 \mathrm{kPa}$ ) of the coin, showing the finer structure. The magnified image shows the letters "RTY" of the word "LIBERTY" on the coin. (E) and (F) Comparison between the topographic and $I_{\mathrm{EL}}$ scan across letter " $\mathrm{Y}$ " of "LIBERTY." The inset of (F) shows the local roughness of the coin. imaged by mapping $J$ at the intersection points of the top and bottom electrode lines (similar to a display device). Both $I_{\mathrm{EL}}$ and $J$ increase linearly with $\sigma$; however, the sensitivity to bias for an optical device is larger (by a factor of about 3), which implies that the latter has a larger dynamic range (Fig. 4B). For $I_{\mathrm{EL}} \sim 5$ (corresponding to a signal-to-noise ratio of 5) the sensitivity of the optical device is $\sim 9 \mathrm{kPa}$ at a bias of $25 \mathrm{~V}$. Assuming a minimum measurable current signal of $1 \mathrm{nA}$ for the electronic device proposed in the inset of Fig. 4A, for the intersection point of $\sim 20 \mu \mathrm{m}^{2}$ (36) a sensitivity of $\sim 10 \mathrm{kPa}$ would be possible. Because the resolution of $20 \mu \mathrm{m}$ is comparable to the CCD pixel size used in the optical device, a spatial resolution of $\sim 50 \mu \mathrm{m}$ at a sensitivity of $10 \mathrm{kPa}$ should be possible for the electronic device.

There are two practical advantages of the described tunneling-based device compared with the reported tactile imaging devices based on deformable membranes or stress-sensitive materials. First, we achieve high sensitivity
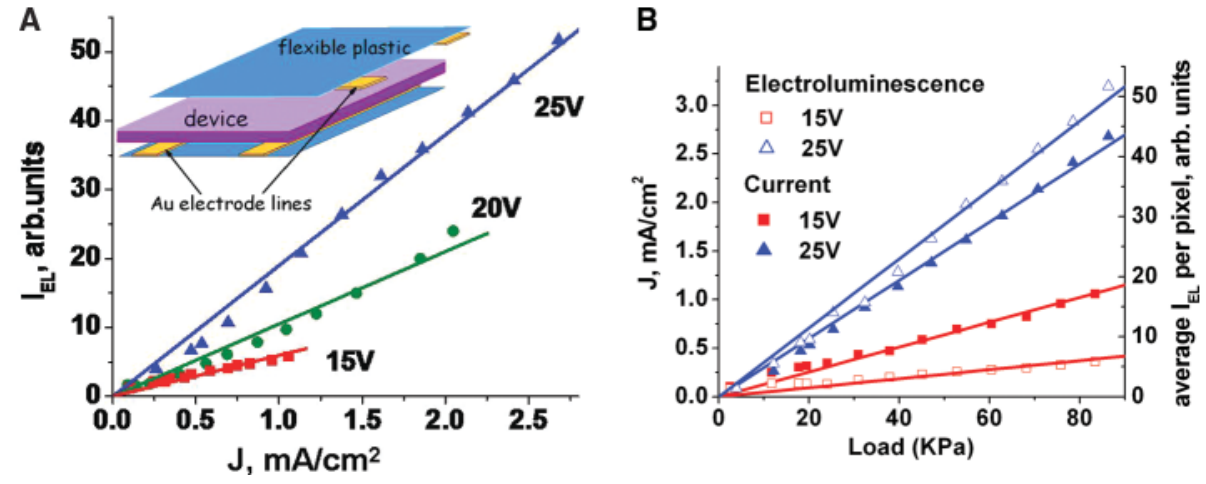

Fig. 4. Comparison of electronic (i.e., current) and optical (i.e., electroluminescence) signals. (A) $I_{\mathrm{EL}}$ is linearly proportional to $\mathrm{J}$. Each data point is at fixed bias with varying $\sigma$. The slope at the three biases are $6.0,10.5$, and 19.0 (arbitrary units) $\mathrm{cm}^{2} / \mathrm{mA}$. The efficiency of the device to convert current to photoluminescence increases with bias as indicated by the slope. (B) Both $J$ and $I_{\mathrm{EL}}$ increase linearly with $\sigma$ (i.e., load) for the 15 to $25 \mathrm{~V}$ bias range studied. The change in slope as bias increases from 15 to $25 \mathrm{~V}$ for $J$ and $I_{\mathrm{EL}}$ is 2.34 and 7.63 , respectively.

because tunneling depends exponentially on displacement (i.e., strain). Second, the selfassembly process involves no complex lithog- raphy, making it easier to build the device directly on surfaces of large area and complex shapes. 


\section{References and Notes}

1. G. Robles-De-La-Torre, V. Hayward, Nature 412, 445 (2001).

2. S. Matsumoto et al., Surg. Endosc. Ultrasound Intervent. Tech. 11, 939 (1997).

3. N. Sakai, M. Tatsuta, H. Yano, H. lishi, S. Ishiguro, Gastrointest. Endosc. 51, 69 (2000).

4. O. Tohyama, S. Maeda, H. Itoh, IEEE J. Sel. Top. Quant. Electron. 5, 115 (1999).

5. P. K. Plinkert, I. Baumann, E. Flemming, Laryngorhinootologie 76, 543 (1997)

6. P. N. Brett, R. S. Stone, Proc. Inst. Mech. Eng. [H.] 211 309 (1997).

7. The tactile signal may be displayed on a monitor as a digital hardness score (2), or a virtual reality tool similar to the NanoManipulator (37) may be developed. In the NanoManipulator, the force from the manipulator performing nanoscale displacement of molecules is transmitted to a joy stick, giving the operator a "touch sensation" during the manipulation

8. P. Dario, E. Guglielmelli, C. Laschi, J. Robot. Syst. 18, 673 (2001).

9. Y. Okumura et al., Adv. Robot. 18, 699 (2004).

10. Y. B. Jia, IEEE Trans. Robot. 21, 726 (2005)

11. M. Kaneko, K. Tanie, IEEE Trans. Rob. Autom. 10, 355 (1994).

12. A. M. Okamura, M. R. Cutkosky, Int. J. Robot. Res. 20 925 (2001).

13. R. Tajima, S. Kagami, M. Inaba, H. Inoue, Adv. Robot. 16, 381 (2002).

14. M. Shikida, T. Shimitzu, K. Sato, K. Itoigawa, Sens. Actuators A Phys. 103, 213 (2003)

15. J. Engel, ]. Chen, C. Liu, J. Micromech. Microeng. 13, 359 (2003).

16. R. D. Howe, M. R. Cutkosky, IEEE Trans. Rob. Autom. 9, 140 (1993).

17. D. De Rossi, F. Carpi, E. P. Scilingo, Adv. Colloid Interface Sci. 116, 165 (2005)

18. C. Domenici, D. Derossi, Sens. Actuators A Phys. 31, 97 (1992).
19. S. P. Lacour, C. Tsay, S. Wagner, IEEE Electron Device Lett. 25, 792 (2004)

20. T. Someya et al., Proc. Natl. Acad. Sci. U.S.A. 101, 9966 (2004).

21. N. J. Ferrier, R. W. Brockett, Int. J. Robot. Res. 19, 795 (2000).

22. B. L. Gray, R. S. Fearing, IEEE Int. Conf. Robot. Autom. 1, 1 (1996).

23. J. W. Morley, A. W. Goodwin, I. Darian-Smith, Exp. Brain Res. 49, 291 (1983)

24. R. S. Johansson, R. H. LaMotte, Somatosens. Res. 1, 21 (1983)

25. As a finger touches a surface, the fingernail color begins to redden above a threshold force of $\sim 0.3 \mathrm{~N}$ and completely reddens at $\sim 1 \mathrm{~N}$. For ordinary grabbing and sensing operations, the force at the fingertip ranges from 1 to $4 \mathrm{~N}$. Assuming a contact area of $\sim 1 \mathrm{~cm}^{2}$, a reasonable threshold stress to determine texture by human finger would be in the 10 to $40 \mathrm{kPa}$ range (38).

26. G. Decher, Science 277, 1232 (1997).

27. C. Y. Jiang, S. Markutsya, Y. Pikus, V. V. Tsukruk, Nat. Mater. 3, 721 (2004)

28. Alternating monolayers of $\mathrm{Au}(10 \mathrm{~nm}$ ) and CdS ( 3 to 4 $\mathrm{nm}$ ) nanoparticles (NP), spaced by a dielectric layer (DL) composed of PSS and PAH, are deposited using a layerby-layer assembly process by sequentially dipping (26) in four solutions: The $0.1 \%$ (by weight) solutions of PSS and PAH of molecular weights 70 and $15 \mathrm{kD}$ have a pH of 8 and 4.2 , respectively. The anionic Au and CdS deposition solutions with $10^{12}$ and $10^{16}$ particles/ml have a pH of 6 and 4.5 , respectively. The deposition time for the polymer monolayer is 30 min each; for the Au and cationic CdS nanoparticles, it is 24 hours and 1 hour, respectively. All the solutions are in DI water (filtered through $20 \mu$ Millipore filter), and each deposition step is followed by a rigorous wash in DI. The chemical structure of DL between Au and CdS nanoparticle monolayers is Au(NP)/PAH/(PSS/PAH)/PSS/ $\mathrm{CdS}(\mathrm{NP})$. The process is initiated by cleaning the 1-inch by 1-inch ITO-coated (400 nm) glass surface in piranha solution $\left(\mathrm{H}_{2} \mathrm{SO}_{4} / \mathrm{H}_{2} \mathrm{O}_{2} ; 3: 1\right.$ by volume) to make the electrode surface negatively charged. The final structure of the device

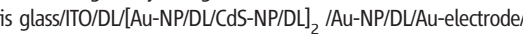
plastic. The structure of the flexible electrode is $\mathrm{Au}(200 \mathrm{~nm}) / \mathrm{Cr}$ $(70 \mathrm{~nm}) / \mathrm{Al}(12 \mu \mathrm{m}) / \mathrm{siloxane}$ rubber $(\sim 5 \mu \mathrm{m})$. The Au is sputter-deposited on the Al foil with $\mathrm{Cr}$ as the adhesion layer. Poly(dimethyl siloxane) is spincast on the other side for the electrode, followed by UV cross-linking. The resultant freestanding electrode is physically placed on the device with the Au side in physical contact with the device surface.

29. R. H. Fowler, L. Nordheim, Proc. R. Soc. London A 119, 173 (1928).

30. T. Cassagneau, T. E. Mallouk, J. H. Fendler, J. Am. Chem. Soc. 120, 7848 (1998).

31. J. Y. Ouyang, C. W. Chu, C. R. Szmanda, L. P. Ma, Y. Yang, Nat. Mater. 3, 918 (2004).

32. S. O. Kasap, in Principles of Electrical Engineering Materials and Devices, Revised Edition, S. O. Kasap, Ed. (McGraw Hill, New York, 2000), pp. 284-288.

33. D. M. DeLongchamp, P. T. Hammond, Chem. Mater. 15 , 1165 (2003).

34. M. F. Durstock, M. F. Rubner, Langmuir 17, 7865 (2001).

35. K. L. Johnson, Contact Mechanics (Cambridge Univ. Press, Cambridge, 1985)

36. Recently, 20- $\mu \mathrm{m}$ device features have been demonstrated on flexible circuits (39).

37. A. Seeger et al., J. Vac. Sci. Technol. B 19, 2717 (2001)

38. S. A. Mascaro, H. H. Asada, IEEE Trans. Robot. Autom. 17, 698 (2001)

39. T. W. Kelley et al., Chem. Mater. 16, 4413 (2004)

40. We thank the Office of Naval Research (N00014-01-10977) and the National Science Foundation (534812) for financial support.

14 February 2006; accepted 7 April 2006 10.1126/science. 1126216 